\title{
BioSounds: an open-source, online platform for ecoacoustics
}

\section{[version 1; peer review: 2 approved]}

\author{
Kevin F.A. Darras (D1* ${ }^{*}$, Noemí Pérez ${ }^{1 *}$, Mauladi -2 , Tara Hanf-Dressler ${ }^{1}$ \\ ${ }_{1}^{1}$ Agroecology, University of Göttingen, Göttingen, Niedersachsen, 37077, Germany \\ ${ }^{2}$ Department of Information Systems, Universitas Jambi, Jambi, Jambi, 36122, Indonesia \\ * Equal contributors
}

V1 First published: 12 Oct 2020, 9:1224

https://doi.org/10.12688/f1000research.26369.1

Latest published: 01 Mar 2023, 9:1224

https://doi.org/10.12688/f1000research.26369.2

\begin{abstract}
Passive acoustic monitoring of soundscapes and biodiversity produces vast amounts of audio recordings. However, the management of these raw data presents technical challenges and their analysis suffers from bottlenecks. A multitude of software solutions exist, but none can perform all the data processing needed by ecologists for analysing large acoustic data sets. The field of ecoacoustics needs a software tool that is free, evolving, and accessible. We take a step in that direction and present BioSounds: an open-source, online platform for ecoacoustics designed by ecologists and built by software engineers. Biosounds can be used for archiving and sharing recordings, manually creating and reviewing annotations of sonant animals in soundscapes, analysing audio in time and frequency, and storing reference recordings for different taxa. We present its features and structure, and compare it with similar software. We describe its operation mode and the workflow for typical use cases such as the analysis of bird and bat communities sampled in soundscape recordings. BioSounds is available from:

https://github.com/nperezg/biosounds
\end{abstract}

\section{Keywords}

Soundscape, sound analysis, ecoacoustics, passive acoustic monitoring, automated sound recording, autonomous recording units, spectrogram, audio annotation

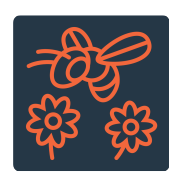

This article is included in the Ecology and

Global Change gateway.

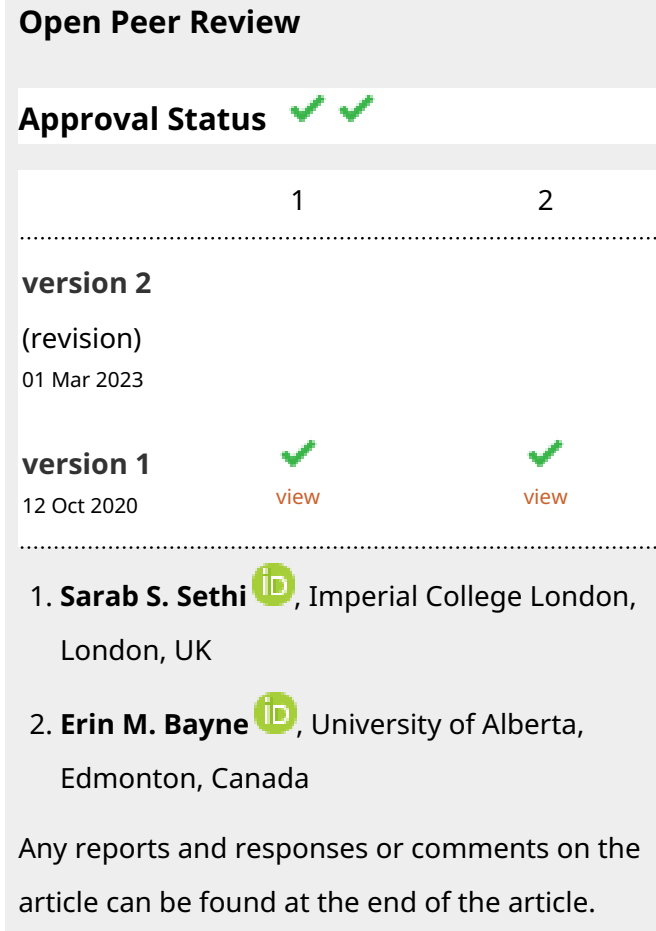

1 2

version 2

(revision)

01 Mar 2023

version 1

12 Oct 2020

1. Sarab S. Sethi ID, Imperial College London,

London, UK

2. Erin M. Bayne ID, University of Alberta,

Edmonton, Canada

Any reports and responses or comments on the article can be found at the end of the article. 
Corresponding authors: Kevin F.A. Darras (kdarras@gwdg.de), Noemí Pérez (noemi@crazycodelady.com)

Author roles: Darras KFA: Conceptualization, Data Curation, Formal Analysis, Investigation, Methodology, Project Administration, Software, Supervision, Validation, Visualization, Writing - Original Draft Preparation, Writing - Review \& Editing; Pérez N: Investigation, Methodology, Project Administration, Resources, Software, Supervision, Validation, Visualization, Writing - Original Draft Preparation, Writing - Review \& Editing; - M: Investigation, Methodology, Software, Writing - Review \& Editing; Hanf-Dressler T: Investigation, Methodology, Validation, Writing - Review \& Editing

Competing interests: No competing interests were disclosed.

Grant information: This work was funded by the Deutsche Forschungsgemeinschaft, grants number SFB990/1 and SFB990/2. The funders had no role in study design, data collection and analysis, decision to publish, or preparation of the manuscript.

Copyright: $\odot 2020$ Darras KFA et al. This is an open access article distributed under the terms of the Creative Commons Attribution License, which permits unrestricted use, distribution, and reproduction in any medium, provided the original work is properly cited.

How to cite this article: Darras KFA, Pérez N, - M and Hanf-Dressler T. BioSounds: an open-source, online platform for ecoacoustics [version 1; peer review: 2 approved] F1000Research 2020, 9:1224 https://doi.org/10.12688/f1000research.26369.1

First published: 12 Oct 2020, 9:1224 https://doi.org/10.12688/f1000research.26369.1 


\section{Introduction}

Automated, passive recording for biodiversity research has come of age. It presents new opportunities for ecologists, but yields huge amounts of data that are challenging to manage ${ }^{1}$. The resulting recordings are raw data that require considerable effort to extract the ecological information contained within. To realise the potential of ecoacoustics projects, different software tools are required in different data processing and analysis stages: First, soundscape recordings - comprising all sounds recorded in a landscape ${ }^{2}$ - need to be archived and made accessible to collaborators, either locally or remotely ${ }^{3}$. Sometimes, an optional pre-processing stage (e.g., re-sampling, merging and splitting, compressing of recordings) is conducted using audio editing software ${ }^{4}$. In general, recordings need to be visualized using spectrograms (i.e., sonograms) and played back to detect, identify, and then manually annotate the target sounds (usually sonant animal species) ${ }^{4,5}$. To our knowledge, no dedicated tool allows for a consistent, structured validation workflow of these manual annotations yet by independent experts. Increasingly, automated detection and classification of sounds is used to facilitate processing large amounts of audio data ${ }^{6}$. The latter still need to be verified by human experts, who rely either on their own knowledge, or reference recordings found in audio repositories (i.e., reference sound libraries) linking recordings to species identi$\operatorname{ties}^{7}$. Alternatively to their time-consuming manual annotation, soundscapes can be characterised with automatically computed eco-acoustic indices that can be linked to biodiversity metrics $^{8,9}$, or with general acoustic feature sets that can be used to detect anomalous sound events in an unsupervised manner ${ }^{10}$. Finally, in bioacoustics- or behavior-focused studies, but also for the identification of bats, the target sounds need to be analysed further by measuring their properties in the frequency-timeamplitude space $^{11,12}$. At the time of writing, no software integrates all these different data processing stages into a consistent, integrated workflow, and reference libraries are still scarce for particular species groups ${ }^{1}$.

Software tools that handle audio data need to be built sustainably to benefit a large user base in the research community. While the majority of software is free, few are online-based, many are specialised on specific taxa, and only half of them are open-source (Table 1). It is essential to have free tools that all researchers and practitioners can use, irrespective of their budget constraints. Also, only open-source projects guarantee that they can be continuously developed to keep up with the pace of technological progress, that they stay accessible over time, and that the actual functions are transparent and replicable. Within two years, three out of the 19 reported software tools by Priyadarshani et al. ${ }^{6}$ appear to have ceased development. Accessibility, which is essential for international collaboration and verification of bioacoustic data ${ }^{13}$, also requires online solutions that are mostly independent of operating systems or any commercial software. In a nutshell, the field of ecoacoustics requires an open-source, online tool, as this fulfils most requirements: being free, easily maintainable, collaborative and accessible.
We present BioSounds: an open-source, online platform for ecoacoustics, designed by ecologists and built by software engineers. Currently, BioSounds can be used to manage soundscape and reference recording collections, to manually create and independently review annotations in recordings, and to perform basic sound measurements in time and frequency. BioSounds was originally based on Pumilio ${ }^{3}$ but the latter has ceased development and Biosounds has considerably expanded since. At the moment of writing, only one other software - Ecosounds - offers similar functions as BioSounds ${ }^{5}$, and we compare them with each other (Table 2). We detail the structure and functionality of BioSounds in the following and announce our development goals.

\section{Methods}

\section{Implementation}

Coding languages, libraries, and tools. BioSounds is a webbased application written in PHP $7^{14}$, Python $2.7^{15}$, Javascript ${ }^{16}$, JQuery $3.4^{17}$, Twig $2^{18}, \mathrm{CSS}^{19}$ and HTML $5^{20}$. It uses Web Audio $\mathrm{API}^{21}$, Sox $14.4^{22}$, Lame ${ }^{23}$ and ImageMagick ${ }^{24}$ software for sound and image processing, a MySQL ${ }^{25}$ database for organising the data (Figure 1), a RabbitMQ ${ }^{26}$ queue for file processing, Plupload 1.5 as a visual file upload tool ${ }^{27}$, JQuery UI $1.12^{28}$, JCrop $0.9^{29}$, Bootstrap $4.3^{30}$ and the Symfony 4 process component ${ }^{31}$ for managing the scripts execution. The Python libraries used are: $\mathrm{Numpy}^{32}$, Pillow ${ }^{33}$ and Audiolab $0.8^{34}$. We containerized the project using Docker ${ }^{35}$, which spares software developers the time for installing libraries, the database, and configuring the server. This setup allows developers to run the project on their machines quickly and free of typical installation issues like library version incompatibilities.

Audio visualization and playback. The core sound visualisation and playback tasks are handled by two distinct components. First, spectrogram images are generated by the Python script 'sound visualization tool', which was created for the discontinued 'Pumilio' project ${ }^{3}$. This script generates spectrograms by computing a Fast-Fourier Transform on the waveform of the audio recording. Second, sound playback and speed control use Web Audio API, a high-level application programming interface for processing and synthesizing audio in web applications. It is included in modern browsers to take advantage of the browser resources without requiring any extra media player or library in our project, and we plan to use it for generating spectrograms too.

\section{Operation}

Server installation. BioSounds is published in a GitHub repository ${ }^{36}$ and needs to be installed in a web server to run. Instructions and general information regarding the setup for developers and the production server are included in the README file on GitHub. The BioSounds installation for local development (in the developer's machine) is facilitated by a Docker setup. We provide a set of Docker configuration files that can also aid the server installation, but the final setup should be carried out by the server administrator (or devOps 


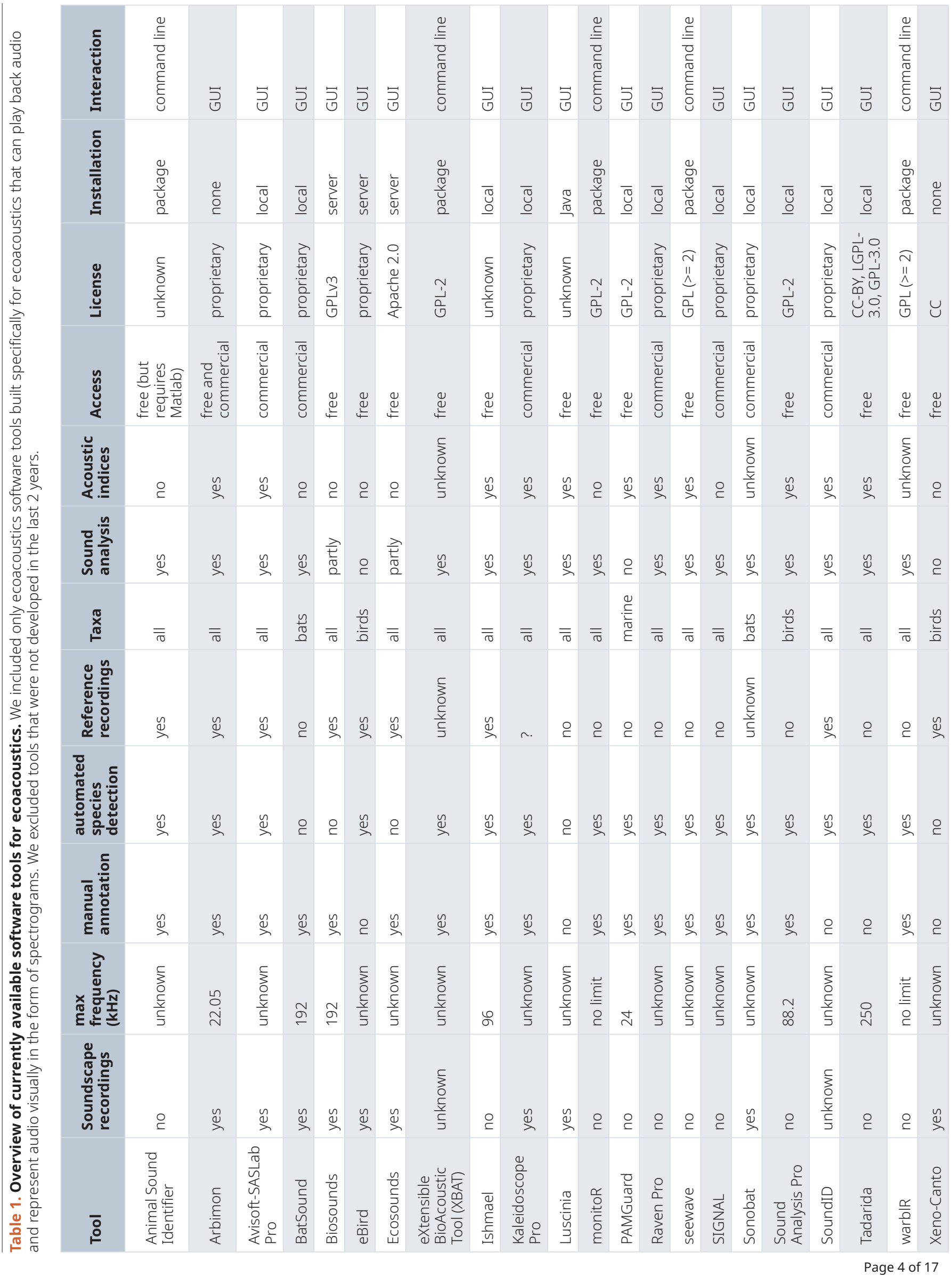


Table 2. Differences between BioSounds and Ecosounds. We compared both software tools' functionalities that are relevant for administrators and normal users. Both tools work with Chrome, Firefox, and Internet Explorer browsers.

\begin{tabular}{|c|c|c|c|}
\hline Category & Criteria & Biosounds & Ecosounds \\
\hline \multirow[t]{8}{*}{ Management } & Accessibility & $\begin{array}{l}\text { Open collections available. Users } \\
\text { registered by administrators on demand. } \\
\text { Users can access all collections. }\end{array}$ & $\begin{array}{l}\text { Open collections available. Self-registration of users. } \\
\text { Project owners can define user access. }\end{array}$ \\
\hline & Organisation & $\begin{array}{l}\text { Creation of collections by administrator } \\
\text { via database. Option for collection } \\
\text { description. }\end{array}$ & $\begin{array}{l}\text { Creation of projects by user via website interface. } \\
\text { Option for project image, description, and location } \\
\text { (visible on map). }\end{array}$ \\
\hline & Recordings & $\begin{array}{l}\text { Administrators can upload, delete, rename } \\
\text { sound recordings. Recording size limit: } \\
300 \text { MB. }\end{array}$ & $\begin{array}{l}\text { No upload of audio files directly through website } \\
\text { possible; manual inspection of data and quality } \\
\text { control by main administrator. Recording limit: from } \\
1 \text { hour to } 1 \text { day }(>1 \mathrm{~GB})\end{array}$ \\
\hline & $\begin{array}{l}\text { Reference } \\
\text { collection }\end{array}$ & $\begin{array}{l}\text { Reference collections available with } \\
\text { dedicated list view. Tags can be marked as } \\
\text { reference. }\end{array}$ & $\begin{array}{l}\text { Annotation library of animal sounds in extra } \\
\text { sub-menu available (1390 recordings). Tags in } \\
\text { spectrograms can be marked as reference. }\end{array}$ \\
\hline & $\begin{array}{l}\text { Long } \\
\text { soundscapes }\end{array}$ & $\begin{array}{l}\text { Spectrogram range limited by } 300 \text { MB file } \\
\text { size limit. }\end{array}$ & $\begin{array}{l}\text { Multiple recordings can be displayed on large } \\
\text { temporal scales across project sites. }\end{array}$ \\
\hline & Statistics & $\begin{array}{l}\text { Overview of users accessible to } \\
\text { administrator. }\end{array}$ & $\begin{array}{l}\text { Statistics of annotations, projects, sites, recordings, } \\
\text { users, etc. can be displayed. }\end{array}$ \\
\hline & Playback duration & $\begin{array}{l}\text { Logged for each user and recording, } \\
\text { accessible to admin in database. }\end{array}$ & not available \\
\hline & Guide & Online public user guide & not available \\
\hline \multirow[t]{6}{*}{ Annotation } & Database & $\begin{array}{l}\text { Species names of birds, bats and frogs, } \\
\text { mainly of Southeast Asia, can be entered } \\
\text { in Latin or English. Species list can be } \\
\text { expanded by admins in database. }\end{array}$ & $\begin{array}{l}\text { Species names and of birds, frogs, mammals and } \\
\text { other sounds, mainly from Australia, can be entered } \\
\text { in Latin or English. }\end{array}$ \\
\hline & Creation & $\begin{array}{l}\text { Creation of new annotation (tag) via } \\
\text { spectrogram selection and button click. }\end{array}$ & $\begin{array}{l}\text { Annotation automatically created via spectrogram } \\
\text { selection. }\end{array}$ \\
\hline & Editing & $\begin{array}{l}\text { Editing, deleting of annotation in pop-up } \\
\text { window. Option to estimate call distance. } \\
\text { No option to copy or move tags. }\end{array}$ & $\begin{array}{l}\text { Editing and deleting of annotation in main window. } \\
\text { No option to copy or move tags. }\end{array}$ \\
\hline & Review & $\begin{array}{l}\text { Dedicated function for species validation } \\
\text { by users with reviewing privileges. } \\
\text { Annotations can be zoomed. }\end{array}$ & not available \\
\hline & Identification & $\begin{array}{l}\text { Direct link to Google images and Xeno- } \\
\text { Canto to check species identification. }\end{array}$ & not available \\
\hline & Download & $\begin{array}{l}\text { Download by administrator through } \\
\text { database. }\end{array}$ & Download in CSV by user through website interface. \\
\hline \multirow[t]{7}{*}{ Recording } & Playback & $\begin{array}{l}\text { Play/pause, stop button below } \\
\text { spectrogram. Cursor can be dragged. } \\
\text { Option for continuous playback. }\end{array}$ & $\begin{array}{l}\text { Play/pause button below spectrogram. Cursor can } \\
\text { be dragged. Option to rewind, fast forward and } \\
\text { rewind recording ( } 30 \mathrm{~s}) \text {. }\end{array}$ \\
\hline & Analysis & $\begin{array}{l}\text { Time and frequency coordinates displayed } \\
\text { for current spectrogram or selection, can } \\
\text { be exported to clipboard via button. }\end{array}$ & $\begin{array}{l}\text { Time displayed below spectrogram. Frequency only } \\
\text { displayed for selections. }\end{array}$ \\
\hline & Ultrasound & $\begin{array}{l}\text { Up to } 192 \mathrm{kHz} \text { in Chrome and Firefox. } \\
\text { Playback speed can be adjusted between } \\
0.05 \text { and } 1 \mathrm{x} \text {. }\end{array}$ & not available \\
\hline & Filtering & $\begin{array}{l}\text { Filtering of sound frequencies outside } \\
\text { zoomed selection, checked by default } \\
\text { (what you see is what you hear). }\end{array}$ & not available \\
\hline & Navigation & $\begin{array}{l}\text { Any part of spectrogram in time and } \\
\text { frequency can be navigated to and } \\
\text { zoomed into. }\end{array}$ & Only navigation along time axis. \\
\hline & Visibility & $\begin{array}{l}\text { Left and right audio channels can be } \\
\text { displayed separately for stereo recordings. } \\
\text { FFT window size set by administrator } \\
\text { through website interface. }\end{array}$ & $\begin{array}{l}\text { No option to change audio channel or FFT window } \\
\text { size. }\end{array}$ \\
\hline & Download & $\begin{array}{l}\text { Recording: MP3 or OGG (ultrasound). } \\
\text { Spectrogram: PNG }\end{array}$ & Recording: WAV or MP3. Spectrogram: PNG \\
\hline
\end{tabular}




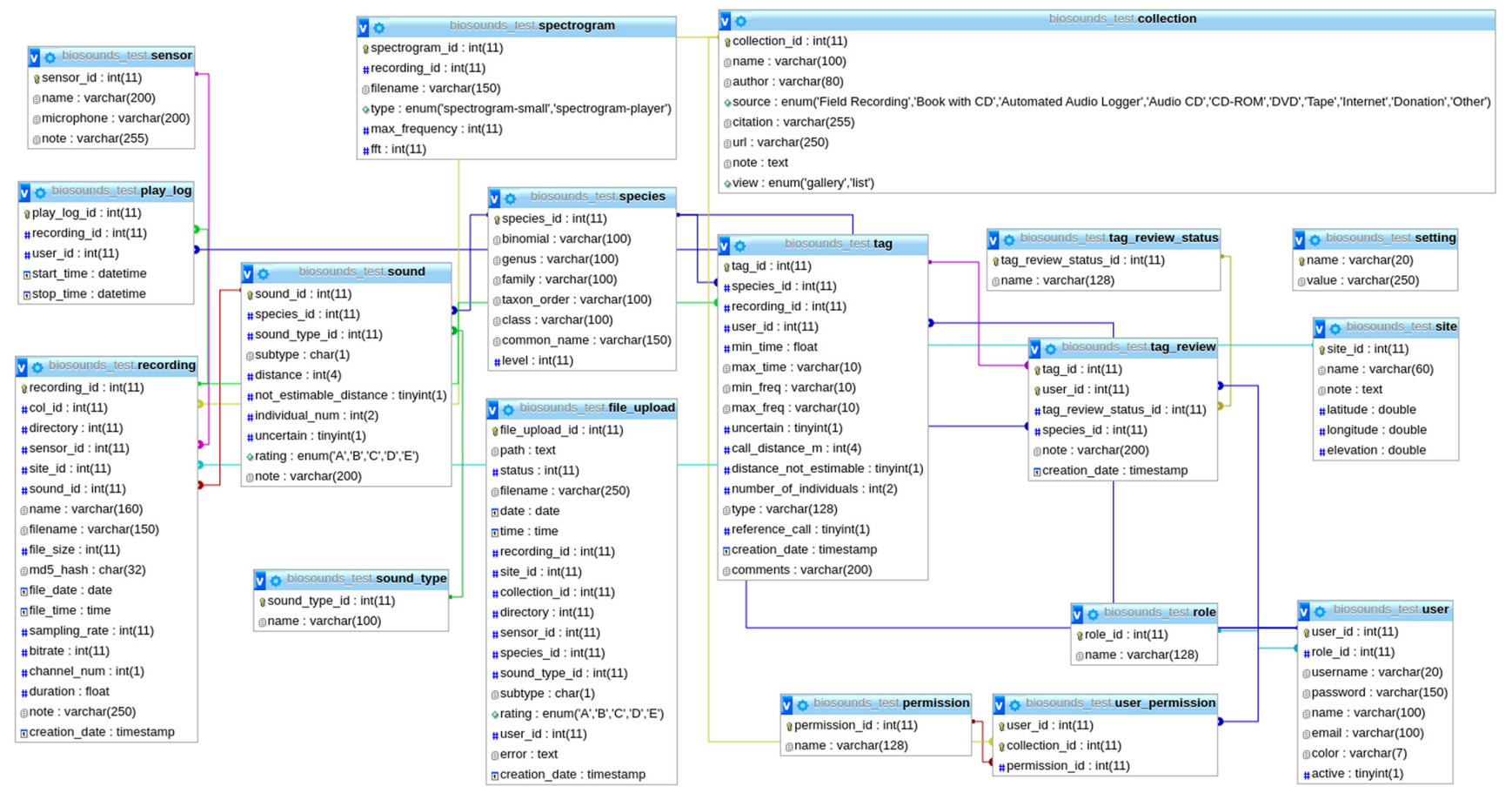

Figure 1. MySQL database structure in BioSounds.

engineer) of the institution. For server installations without Docker, a step-by-step installation guide is provided in the repository.

Access. We run an online instance of BioSounds for our project SoundEFForTS ${ }^{37}$, where most of the steps described in the use cases below can be reproduced. This website can host public reference collections (i.e., reference audio libraries) for prospective users, for instance for Chiroptera and Anura. Soundscape collections, due to their larger size, can be integrated up to a manageable size for projects contributing to BioSounds development.

Users can access BioSounds (both the existing instance and future installations) via a desktop browser with an internet connection. BioSounds works with Windows, Linux, and MacOS operating systems and the most common internet browsers (Firefox, Chrome, Safari).

Collections. BioSounds organises audio recordings (named "recordings" hereafter) within collections. Collections can be accessed through the "Collections" drop-down menu. Those that are part of ongoing research projects are only visible to registered users; open collections are public. Collection creation is still handled directly via the database by adding it to the table 'Collection'. Administrators can then upload recordings in most common audio formats into collections. PNG image previews of the spectrograms and MP3s of the audio file are generated after insertion into the database. Audio recordings can be given names that differ from the default file name. Collections can be shown with a gallery view (thumbnails with sound names) or a list view with larger spectrograms and a simple audio player, and comments can be inserted. There are two types of collections in BioSounds: soundscape recording collections ("soundscape collections" hereafter) and reference recording collections (i.e., reference audio libraries; named "reference collections" hereafter).

Soundscape collections contain field recordings which each encompass a range of sounds from a particular site during a particular time interval. The recordings within are displayed with the gallery view by default, which shows either mono or stereo thumbnails of their spectrograms along with the sound names and the overlaid maximum recorded sound frequency (Figure 2).

Reference recording collections link individual recordings to identified sound sources (typically, sonant animal species). They display recordings with a list view by default; an example is shown in the public "Reference collection Anura". Reference collections can host recordings that are needed for supporting the identification of the animals of particular taxa or regions. The spectrogram in the list view gives a rapid overview of the reference sound, and the embedded audio player can play it back directly (Figure 3). In contrast to soundscape recordings, uploaded reference recordings need to be assigned to animal species, and they can have a vocalisation type and quality rating. Reference collections can be filtered by species and rating. 
Notes: ID by Novia, reviewed by Andres

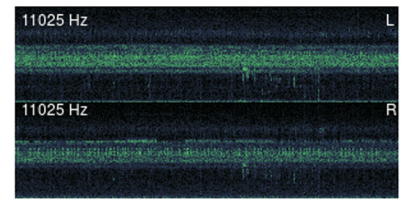

Demonstration recording

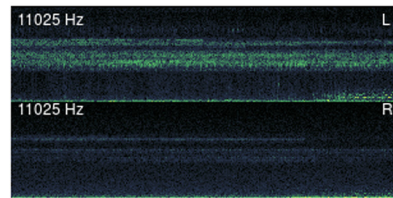

P01_US2_20170330_175951.flac

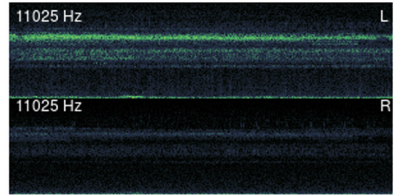

P01_US2_20170329_180012.flac

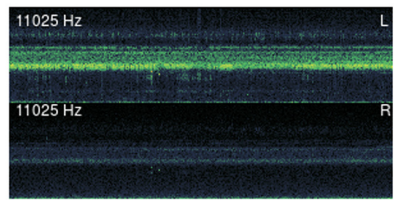

P01_US2_20170331_060905.flac

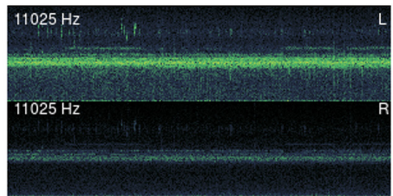

P01_US2_20170330_070920.flac

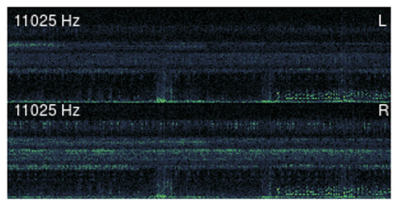

P02_US5_20170317_180424.flac

Figure 2. The default gallery view for soundscape recording collections in BioSounds.

\title{
Reference collection Anura 15 sounds
}

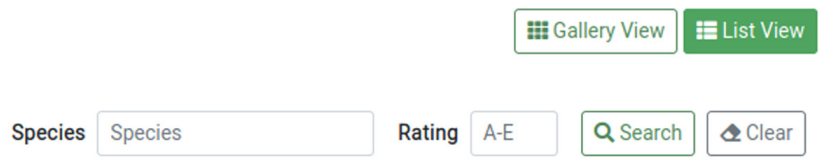

\author{
Amnirana nicobariensis \\ Type: song \\ Subtype: \\ Rating: \\ Filename: Amnirana nicobariensis \\ Date: $24 / 05 / 2017$ \\ Time: 18:20:00
}

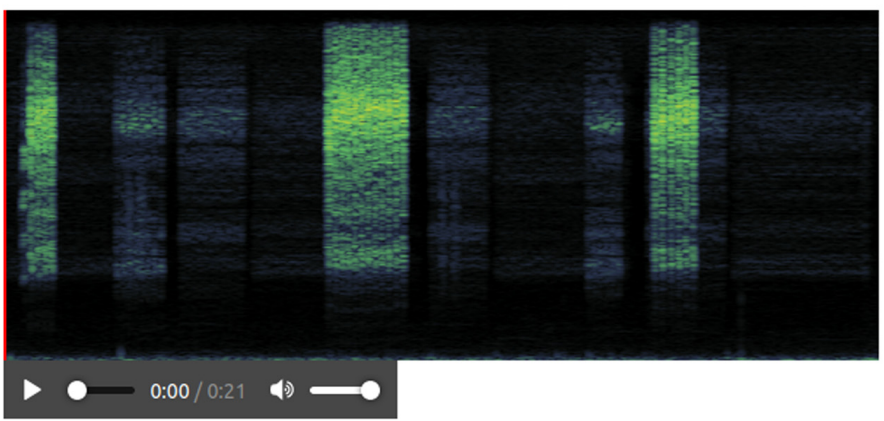

Figure 3. Reference recording shown in list view. Reference collections can be filtered by species and rating, and additional information regarding the recording is displayed. Reference recordings can be played back directly in list view.

Users. BioSounds has two registered user classes: normal users and administrators. All registered users can open all recordings inside the spectrogram player, as well as create, view, and edit their own annotations (called "tags" in BioSounds) that are linked to sound sources (mostly sonant animal species). Normal users have viewing and reviewing privileges for other users' tags that are set by administrators for single collections. Administrators can view, review, and edit all users' tags. They can also create users, set their tags' color, and define their status (normal user/administrator). Finally, administrators can upload, rename, and delete recordings.

Spectrogram player. Recordings can be opened in the spectrogram player (Figure 4). Spectrograms are visualisations of sound where sound amplitude is shown in color or grayscale tones, time is shown on the $\mathrm{X}$ axis, and frequency is displayed on the 


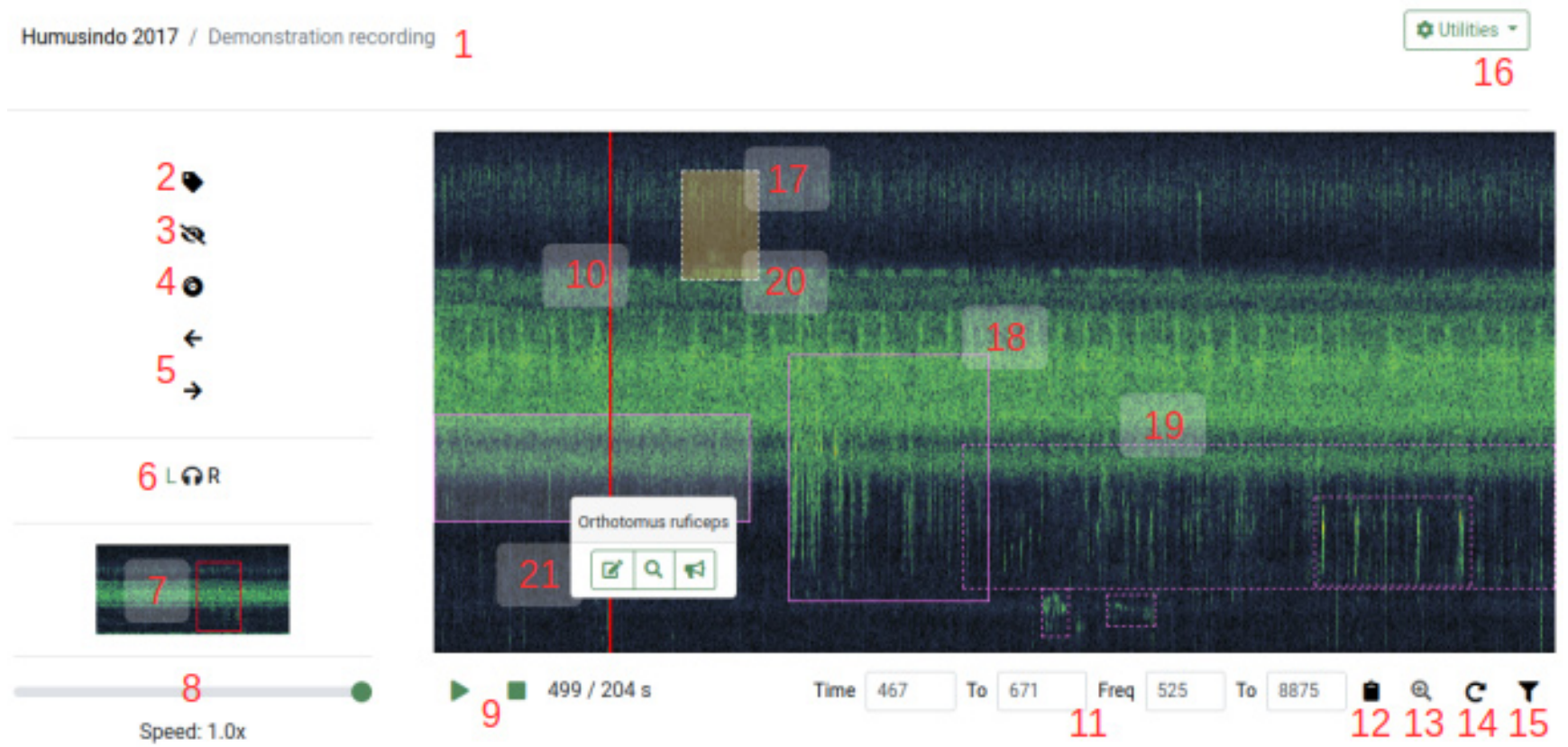

Figure 4. BioSounds spectrogram player. 1: sound and collection name. 2: annotating/tagging sounds. 3: hiding/showing tags. 4: playback mode. 5: moving window left and right. 6: audio channel selection. 7: overview spectrogram, red rectangle shows current view. 8: playback speed. 9: playback/pause and stop, time position. 10: playback cursor. 11: time (s) and frequency (Hz) coordinates of current view or selection. 12: copying time and frequency coordinates. 13: zooming. 14: continuous playback. 15: frequency filter. 16: utilities: image and audio download, file info. 17: tags of different users shown with different colors. 18: reviewed tags with solid border. 19: not yet reviewed tags with dashed border. 20: tags without detection distance with orange shading. 21: tag species appears on click, with buttons for editing, zooming, and estimating distance.

$\mathrm{Y}$ axis. The spectrogram player offers various functionalities for tagging sounds: it is possible to play back sound, filter frequencies, navigate the spectrogram, assign selections to animal species (or other sound sources), and perform basic sound analysis.

\section{Use cases}

Bird community analysis

Soundscape recordings can be annotated manually and reviewed by expert ornithologists, as exemplified in the collection "Upland plots dry season 2013". Users can scan recordings visually and aurally using the built-in reading mode, which zooms to a $60 \mathrm{~s}$ long section of the recording, including all frequencies, and enables continuous playback. All avian species can be tagged/annotated based on rectangular spectrogram selections along the frequency and time axes. Species are chosen from the integrated species list, and links to Xeno-canto and Google image searches direct the user to the selected species to support identification (Figure 5). Project-specific reference collections can also be consulted to confirm species identification. Unclear identifications can be marked as uncertain. Coordinates (in time and frequency) are saved automatically based on the boundaries of the selection. Tags can be designated as reference recordings for future inclusion into reference recording collections; comments can be inserted. Tags can be zoomed into and any of the current (filtered or unfiltered) spectrogram views (image or audio) can be downloaded for sharing with collaborators. Distances are estimated in a standardised way using a function that enables full-spectrum viewing and playback of the tags based on a spectrogram of the first $30 \mathrm{~s}$ of the tag. Reference audio recordings of test tones emitted at known distances are needed (see recording "Sound transmission - full spectrum" in Demo collection) to estimate detection distances in an unbiased way ${ }^{38}$.

An advantage of automated acoustic survey data is that they can be validated on multiple levels, yielding accurate datasets ${ }^{39}$. In BioSounds, it is possible to review tags for validating species identification as well as auxiliary tag data. Administrators can grant tag reviewing privileges to users other than the creator. Users with reviewing privileges can either accept species identifications, revise them by suggesting other species, or reject them by marking the annotation for deletion (Figure 5). Administrators can also check the listening durations of each user for each recording to verify that all recordings have been listened to in entirety, and to extract a measure of the sampling intensity. Finally, it is possible to train users by letting them learn from other users' annotations after granting them viewing privileges, and thereafter, to test their performance with already annotated recordings where the annotations are invisible to the user being tested. After the validity checks have been run, administrators can export the 


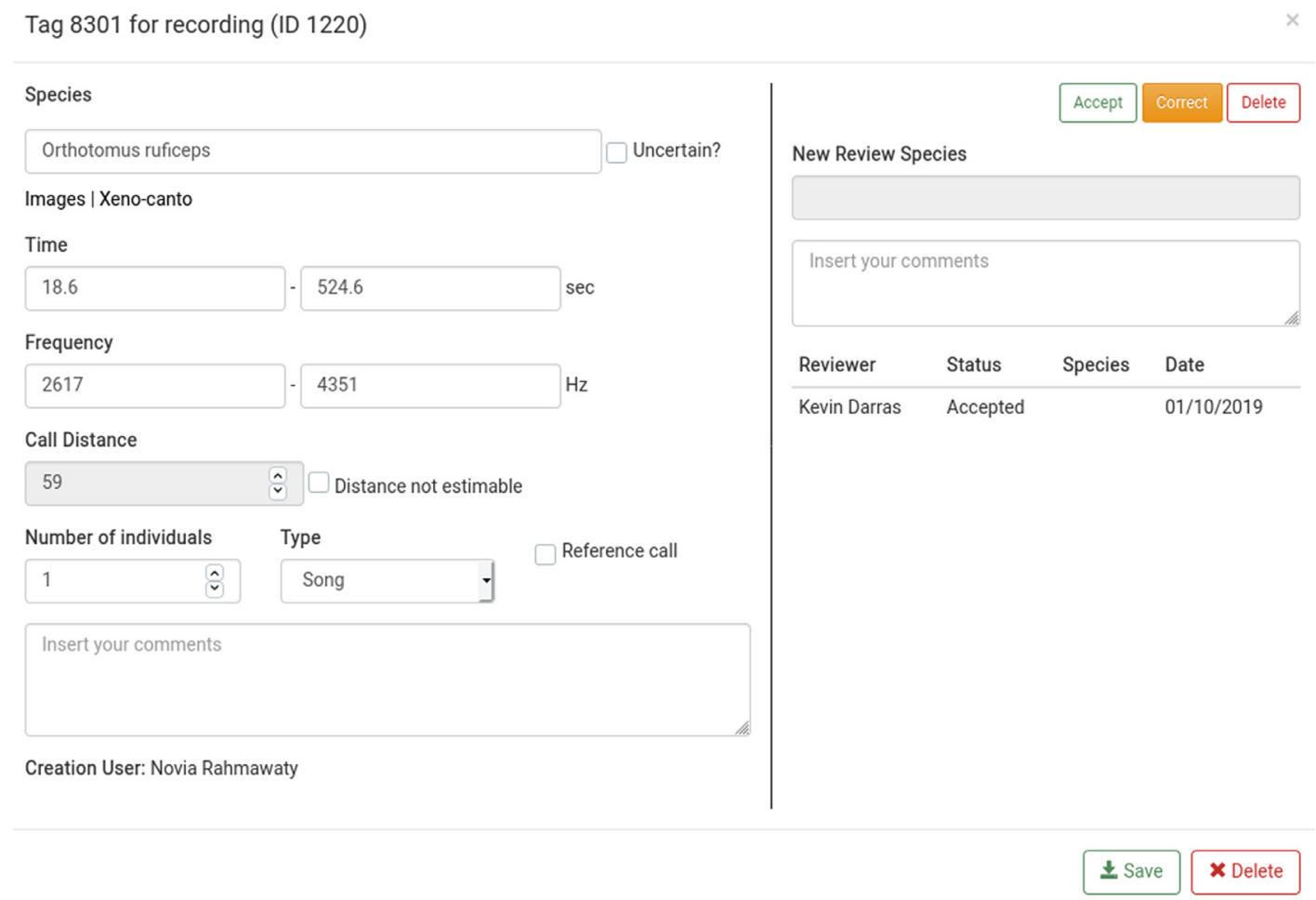

Figure 5. The tag editing window. The right pane is only visible to users with reviewing privileges.

tag data through a MySQL database administration tool like phpMyAdmin $^{40}$ for further statistical analysis.

\section{Bat community analysis}

Soundscape recordings that span the ultrasound frequency range (i.e., ultrasoundscapes) can be similarly analysed with the same functions as for the bird community analysis use case, but they present specific challenges regarding the analysis of bat calls. Most importantly, ultrasound is not audible, so that users need to use the playback speed slider to reduce the playback frequency to artificially hear the ultrasound calls. This can be tested with the annotated example recording "Sample Ultrasoundscape" that is uploaded in the "Demo" collection; any playback rate from 0.05 to 1 can be chosen. To aid in bat call visualisation, the spectrogram settings can also be adjusted by administrators to choose different FFT window sizes.

However, bat species identification is more challenging as bat calls from different species can be similar. Thus, we included bat morphospecies (to be exact, morphocall types) named with single letters from A to $\mathrm{J}$ into the species list, suffixed with digits to designate different call types from the same species. Exact measurement of bat call features (such as start and end frequency, frequency of maximal energy, as well as call and call interval duration) usually determines the assignment bat calls to specific species: using the clipboard button (Figure 4), users can copy the frequency and time coordinates of the current selection to the clipboard to perform basic sound analysis. The exported values can be readily pasted into spreadsheets, and bat call metrics of interest can be rapidly computed with formulae. For those species that have taxonomically unequivocal calls, the users can refer to the reference collection to corroborate their identifications. Finally, manual distance estimation of bat calls is impractical due to their mobility and the fact that we cannot intuitively estimate the distances of human-inaudible sounds, so that the tags can be marked as having not estimable distances.

\section{Conclusions}

BioSounds can be used to archive, visualise, play back, and share soundscape recordings online with users that have different access privileges. The recordings can be analysed collaboratively for detecting sonant animal species such as birds and bats as to derive measures of their activity for use in ecological studies. BioSounds has already been used successfully to analyse bird communities $^{41}$ and to measure bat activities ${ }^{42}$, while performing basic sound analysis. Region- and taxon-specific reference collections can be created, like the anuran calls collection we currently host ${ }^{43}$.

We strive to expand the functionality of BioSounds and keep it accessible in the long term. Open access is a requirement for future development and maintenance. However, it is not a guarantee for a sustainable project either, as some of the open-source projects listed by Priyadarshani et al. in $2018^{6}$ are currently discontinued projects. In Biosounds, we refactored the original Pumilio project, implemented best coding practices, 
and used development tools, like Docker, all of which facilitate developers' work and help them engage in collaboration. We welcome new collaborators to support the project development who could become co-authors on subsequent versions of this article. Among others, we plan to develop the following functions:

- Automated detection and classification of vocalisations, using existing tools $\mathrm{s}^{44,45}$

- Computation of acoustic diversity indices to monitor biodiversity ${ }^{46}$

- Developing the sound analysis tool to include the sound amplitude dimension

- Linking BioSounds to taxonomic databases for an exhaustive, up-to-date list of sonant animals

- Managing and displaying geographic locations of recordings ${ }^{3}$

- Displaying multiple recordings of single sites on a common time axis to visualise longer soundscapes ${ }^{5}$

\section{Data availability}

All the recordings referred to here are accessible in open collections without login on our online instance of BioSounds: https://soundefforts.uni-goettingen.de/biosounds/.

\section{Software availability}

Source code available from: https://github.com/nperezg/biosounds

Archived source code at the time of publication: http://doi. org/10.5281/zenodo. $4047711^{47}$.

License: GNU General Public License v3.0 (GPLv3).

\section{Acknowledgements}

We thank the principal investigators of the B09 sub-project of EFForTS Teja Tscharntke and Ingo Grass for their continued support of BioSounds development through student helper funds, as well as colleagues and students who provided feedback.
1. Gibb R, Browning E, Glover-Kapfer P, et al.: Emerging opportunities and challenges for passive acoustics in ecological assessment and monitoring. Methods Ecol Evol. 2018; 10(2): 169-85. Publisher Full Text

2. Pijanowski BC, Villanueva-Rivera LJ, Dumyahn SL, et al.: Soundscape Ecology: The Science of Sound in the Landscape. BioScience. 2011; 61(3): 203-16. Publisher Full Text

3. Villanueva-Rivera LJ, Pijanowski BC: Pumilio: a web-based management system for ecological recordings. Bull ECOI Soc Am. 2012; 93(1): 71-81. Publisher Full Text

4. Audacity Team: Audacity(R): Free Audio Editor and Recorder. 2018

5. QUT Ecoacoustics Research Group: EcoSounds. GitHub. [cited 2019 Dec 19]. Reference Source

6. Priyadarshani N, Marsland S, Castro I: Automated birdsong recognition in complex acoustic environments: a review. J Avian Biol. 2018; 49(5): jav-01447. Publisher Full Text

7. Xeno-canto Foundation: Xeno-canto: Sharing bird sounds from around the world. Xeno-canto Foundation Amsterdam; 2012.

8. Sueur J, Farina A, Gasc A, et al.: Acoustic Indices for Biodiversity Assessment and Landscape Investigation. Acta Acust United Acust. 2014; 100(4): 772-81. Publisher Full Text

9. Sueur J, Aubin T, Simonis C: Equipment Review-Seewave, A Free Modular Tool for Sound Analysis and Synthesis. Bioacoustics. 2008; 18(2): 213. Reference Source

10. Sethi SS, Jones NS, Fulcher BD, et al: Characterizing soundscapes across diverse ecosystems using a universal acoustic feature set. Proc Natl Acad SCi U S A. 2020 [cited 2020 Jul 8]; 117(29): 17049-17055. PubMed Abstract | Publisher Full Text | Free Full Text

11. The Cornell Lab - Center for Conservation Bioacoustics. Raven Pro.

12. Obrist MK, Boesch R: BatScope manages acoustic recordings, analyses calls, and classifies bat species automatically. CJZ Virtual Spec Issues. 2018; 96(9): 939-54. Publisher Full Text

13. Gaunt SLL, Nelson DA, Dantzker MS, et al.: New Directions for Bioacoustics Collections. The Auk. 2005; 122(3): 984-7. Publisher Full Text

14. PHP: Hypertext Preprocessor. [cited 2020 Aug 24] Reference Source

15. Python Programming Language. Python.org. [cited 2020 Aug 24]. Reference Source
16. JavaScript. MDN Web Docs. [cited 2020 Aug 24]. Reference Source

17. js.foundation JF-. jQuery: Javascript library. [cited 2020 Aug 24]. Reference Source

18. Twig - PHP template engine. Home - Twig - The flexible, fast, and secure PHP template engine. [cited 2020 Aug 24]. Reference Source

19. CSS: Cascading Style Sheets. MDN Web Docs. [cited 2020 Aug 24]. Reference Source

20. HTML5. MDN Web Docs. [cited 2020 Aug 24] Reference Source

21. Web Audio API. MDN Web Docs. [cited 2020 Aug 24]. Reference Source

22. SoX - Sound eXchange | HomePage. [cited 2020 Aug 24]. Reference Source

23. LAME MP3 Encoder. [cited 2020 Aug 24]. Reference Source

24. LLC IS: ImageMagick. ImageMagick. [cited 2020 Aug 24]. Reference Source

25. MySQL. [cited 2020 Aug 24]. Reference Source

26. Messaging that just works - RabbitMQ. [cited 2020 Aug 24]. Reference Source

27. Plupload: Multi-runtime File-Uploader. [cited 2020 Aug 24]. Reference Source

28. js.foundation JF-. jQuery UI. [cited 2020 Aug 24]. Reference Source

29. Hallman K: tapmodo/Jcrop. 2020 [cited 2020 Aug 24]. Reference Source

30. contributors MO Jacob Thornton, and Bootstrap. Bootstrap. [cited 2020 Aug 24]. Reference Source

31. The Process Component (Symfony Docs). [cited 2020 Aug 24]. Reference Source

32. NumPy. [cited 2020 Aug 24]. Reference Source

33. Pillow - Pillow (PIL Fork) 7.2.0 documentation. [cited 2020 Aug 24]. Reference Source

34. Cournapeau D: scikits.audiolab: A python module to make noise from 
numpy arrays. [cited 2020 Aug 24].

Reference Source

35. Empowering App Development for Developers | Docker. [cited 2020 Aug 26].

Reference Source

36. Pérez N: BioSounds. 2020 [cited 2020 Aug 24].

Reference Source

37. BioSounds: SoundEFForTS. [cited 2020 Aug 24] Reference Source

38. Darras K, Furnas B, Fitriawan I, et al.: Estimating bird detection distances in sound recordings for standardizing detection ranges and distance sampling. Methods Ecol Evol. 2018; 9(9): 1928-38. Publisher Full Text

39. Darras K, Batáry P, Furnas BJ, et al.: Autonomous sound recording outperforms human observation for sampling birds: a systematic map and user guide. Ecol Appl. 2019; 29(6): e01954. PubMed Abstract | Publisher Full Text

40. contributors phpMyAdmin: phpMyAdmin. phpMyAdmin. [cited 2020 Aug 22]. Reference Source

41. Darras K, Rahman D, Sugito W, et al.: Birds of primary and secondary forest and shrub habitats in the peat swamp of Berbak National Park, Sumatra [version 2; peer review: 2 approved]. F1000Res. 2018; 7: 229. PubMed Abstract | Publisher Full Text | Free Full Text

42. Denmead LH, Darras K, Clough Y, et al.: The role of ants, birds and bats for ecosystem functions and yield in oil palm plantations. Ecology. 2017; 98(7): 1945-56.

PubMed Abstract | Publisher Full Text

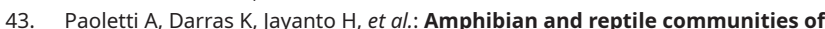
upland and riparian sites across Indonesian oil palm, rubber and forest. Glob Ecol Conserv. 2018; 16: e00492. Publisher Full Text

44. Rhinehart $\mathrm{T}$ : Open source, scalable acoustic classification for ecology and conservation: opensoundscape. kitzeslab; 2019 [cited 2019 Aug 15]. Reference Source

45. Noriega F: Tools for annotating and processing bioacoustic recordings: pylotwhale. 2019 [cited 2019 Aug 15]. Reference Source

46. Buxton RT, McKenna MF, Clapp M, et al.: Efficacy of extracting indices from large-scale acoustic recordings to monitor biodiversity. Conserv Biol. 2018; 32(5): 1174-84

PubMed Abstract | Publisher Full Text | Free Full Text

47. Perez N, Darras K: BioSounds v1.0.0 (Version v1.0.0). Zenodo. 2020. http://www.doi.org/10.5281/zenodo.4047711 


\section{Open Peer Review}

\section{Current Peer Review Status:}

\section{Version 1}

Reviewer Report 20 November 2020

https://doi.org/10.5256/f1000research.29110.r74280

(C) 2020 Bayne E. This is an open access peer review report distributed under the terms of the Creative Commons Attribution License, which permits unrestricted use, distribution, and reproduction in any medium, provided the original work is properly cited.

\section{Erin M. Bayne}

Department of Biological Sciences, University of Alberta, Edmonton, AB, Canada

Biosounds is a very important step forward in the field of bioacoustics. Being able to manage large volumes of digital audio files and the various data that result is a large task that requires "big data" tools. Biosounds is a great step in this direction that continues on from past efforts. I greatly appreciate the efforts the authors have taken to continue developing tools that already existed. Building off of those tools and bringing them together into an easily setup system is a big advance.

A gap in this particular draft of the paper are other similar efforts that do exist. For example, the group I work with has developed Wildtrax (www.wildtrax.ca) that has been operational for several years in Canada. This online system is used by partner agencies in provincial and federal governments, not for profits, industry, and academia. It has similar processes to Biosounds in the area of bioacoustics while also providing a platform for the storage of digital images taken by remote cameras. Including this effort as part of the comparison seems warranted. The key difference is Wildtrax is a centralized database system with the goal of sharing data amongst users Canada-wide rather than have each group setup their own server etc. It too is in development and has many things in common with the list of things Biosounds would like to see developed. The key difference seems to be that we have centralized the process and focused on users bringing their data to one place rather than managing things individually.

While the rationale for WildTrax and Biosounds are different, it does raise what I think is a very important point that would help expand this paper. How do we use tools like WildTrax and Biosounds to create international collaborations and data sharing standards and where might this repository lie? This paper is an excellent description of a wonderful tool but how users that download and setup servers, etc., should consider sharing/using data together is underemphasized. By providing this type of tool Biosounds provides the infrastructure to help us start developing this vision but I feel this paper should also include at least a section for how we should discuss something greater through shared centralized repositories that build off initiatives like Biosounds and WildTrax. Whether a country by country nodal structure is an option that should be discussed rather than having individual labs, researchers, or local government 
departments create their own systems is the next step in determining how we can better utilize the amazing power that autonomous recording devices give us. A discussion of how such a process might be facilitated internationally (i.e. through ornithological societies, bat societies, etc.) would help develop the vision that I think Darras et al. and others envision.

Darras et al must be complemented for developing such a useful tool that will undoubtedly improve the field of bioacoustics immeasurably. It is now up to the bioacoustics and wildlife biology fields to determine the best ways of making this data the most useful for the advancement of science and conservation.

Is the rationale for developing the new software tool clearly explained?

Yes

Is the description of the software tool technically sound?

Yes

Are sufficient details of the code, methods and analysis (if applicable) provided to allow replication of the software development and its use by others?

Yes

Is sufficient information provided to allow interpretation of the expected output datasets and any results generated using the tool?

Yes

Are the conclusions about the tool and its performance adequately supported by the findings presented in the article?

Yes

Competing Interests: My primary comment on this paper is simply that it does not include all platforms that I believe relevant and one of those is a tool I am developing that shares similar functionality.

Reviewer Expertise: I am working in very similar areas trying to achieve a similar objective. My group uses ARUs for a multitude of objectives and needed a tool that could do what is described here. We took a different path than Biosounds but what they have developed is an excellent resource I can see drawing from.

I confirm that I have read this submission and believe that I have an appropriate level of expertise to confirm that it is of an acceptable scientific standard.

Author Response 06 Feb 2023

Kevin Darras

Thank you for your very interesting, complementary appreciation of our work. In the meantime, we have deployed a new version, adding project management, acoustic indices, 
phonies, maps, and timelines to ecoSound-web.

We have discussed the important points you raised in the conclusion with a new paragraph, and we adapted the goals to emphasize inter-operability. These points, together with the need for standards, are also themes in the focus of the first authors' current research projects and interests, which will be expanded further in the next article iteration and website version (c.f. GitHub issues). Note that we already implemented DOI referencing and the choice of Creative Commons licenses for the recordings.

Competing Interests: No competing interests were disclosed.

Reviewer Report 10 November 2020

https://doi.org/10.5256/f1000research.29110.r74102

(C) 2020 Sethi S. This is an open access peer review report distributed under the terms of the Creative Commons Attribution License, which permits unrestricted use, distribution, and reproduction in any medium, provided the original work is properly cited.

\section{Sarab S. Sethi}

Department of Mathematics, Imperial College London, London, UK

In this study Darras et. al present Biosounds, an open source web-based platform for archiving, exploring, and tagging eco-acoustic data. The tool fulfills an important role, and provides ecologists with a user friendly, powerful tool for managing data acquired from projects deploying acoustics.

Whilst the paper was generally well written and clear, my line by line recommendations/comments are as follows:

\section{Introduction}

"19 reported software tools by Priyadarshani" consider rephrasing to "19 software tools reviewed by..." to ensure it is clear that these tools weren't developed by Priyadarshani et. al.

Table 1 presents a comprehensive comparison between Biosounds and other tools. However, it is difficult to parse efficiently in its current format, with a large number of factors to consider, and sorting all the options just alphabetically. Maybe sorting from most to least features, or grouping tools in some way would allow for faster comparisons to be made between the offerings. Additionally, wherever it is placed, putting Biosounds in bold would improve the table.

Table 2, whilst again comprehensive, is arguably not too interesting. Picking out the key few differences and presenting them inline in the text may be a clearer way to present this information - readers are perhaps unlikely to read through the entire page long table. The full table could then be left in supplementary materials 


\section{Methods}

"by computing a fast fourier transform" - what are the parameters for this? And why were they chosen? Are they customisable / do they adapt based on recording parameters?

"Soundscape collections, due to their larger size, can be integrated up to a manageable size" - giving an indicative size in GB/TB is crucial for readers to assess whether this tool is suitable for their own projects.

"upload recordings in most common audio formats" - please detail exactly which formats are supported.

"MP3s of the audio file are generated after insertion into the database" - are the original uploaded audio files retained on the server (e.g., in the case of raw WAV files being uploaded)? Can the users download these back at a later date, or only MP3s? How are the MP3s generated (which codec) and at which compression level?

\section{Use-cases}

"distances are estimated in a standardised way" - how? Using spherical sound absorption assumptions, or by other means? Also, how is distance estimated without knowing the typical source SPL value of a species' call?

"export the tag data through a MySQL database" - are users (not administrators) also able to access this data? It would also be useful to add CSV export option for those not so comfortable with SQL DBs.

"bat morphospecies .... named with single letters from A to J" - you should either detail how these morphospecies differ (what are the identifying call characteristics that places a bat in B for example), or if irrelevant leave out this level of detail and just mention that morphospecies are supported too.

I also had a play around with the live demo running at https://soundefforts.unigoettingen.de/biosounds/. My overall impression was very positive - the site was intuitive to interact with and things generally worked as advertised. I do have a few very minor recommendations below but appreciate that fulfilling these may be more difficult than the suggested manuscript changes:

Opening the player view for a large file can take some time, and it just seems as if the connection has dropped (until finally the page is ready). This is not an issue when going forward and backward within the collection as there is a loading overlay, which makes it clear what is happening.

When loading a large file into the player view the "play" icon is grayed out until some background loading is done. It isn't clear that this is happening until this is done though maybe a loading indicator in place of the gray play icon would be clearer.

Once I'd zoomed in on a part of the spectrogram I couldn't return to the original, zoomedout view without refreshing the page. This should be possible.

When in the player mode there is no indication of sampling frequency or labels on the spectrogram's $Y$ axis. This info can be inferred from the frequency selection box values, but it should be more clearly apparent in my opinion as it is such an important piece of 
information. If an expert is searching for calls from a specific species, frequency labels on the $Y$ axis can make this a lot quicker.

When searching through a collection, if you click on a species from the dropdown prompt, the expected behaviour is that the search will be performed. However, instead a search isn't actually triggered until you click the search button manually.

Is the rationale for developing the new software tool clearly explained?

Yes

Is the description of the software tool technically sound?

Yes

Are sufficient details of the code, methods and analysis (if applicable) provided to allow replication of the software development and its use by others?

Yes

Is sufficient information provided to allow interpretation of the expected output datasets and any results generated using the tool?

Yes

Are the conclusions about the tool and its performance adequately supported by the findings presented in the article?

Yes

Competing Interests: No competing interests were disclosed.

Reviewer Expertise: eco-acoustics, bioacoustics, ecological monitoring, software development

I confirm that I have read this submission and believe that I have an appropriate level of expertise to confirm that it is of an acceptable scientific standard.

Author Response 06 Feb 2023

\section{Kevin Darras}

Thank you for the detailed appraisal of our work. In the meantime, we have deployed a new version (adding project management, acoustic indices, phonies, maps, timelines) and addressed your comments.

Introduction: We removed the ambiguous sentence, which was mentioned in the discussion anyway. ecoSound-web is now in bold, and the Table 1 structure follows the classification of purposes announced in the introduction. Table 2 was deleted.

Methods: We newly implemented FFT window size settings for the users (globally and per recording) and mention the available values in the text. We removed the uninformative "manageable size" and mention the user self-registration feature (which depends on long- 
term funding) in the conclusion's goals. Regarding the SoX audio formats: the list is too long so we prefer to refer to the SoX homepage with a citation, but you can find them at https://sox. sourceforge.net/soxformat.html. We now mention that original audio files are retained on the server, but we don't provide download features yet (see issue \#99 on GitHub).

Use cases: We already provide a citation (10.1111/2041-210X.13031) for the standardised estimation of the detection distances but added some more information to make clear that the reference recordings help human listeners to estimate distances. We now offer CSV export functions for the various data tables (tags, recordings, collections, acoustic indices) that are accessible to users with variable privileges instead of the impractical MySQL access option. Finally, the mention about using letters to distinguish morphospecies (we use "sonotypes" instead) was removed too.

Regarding general usability comments: Spectrogram computation is still time-consuming as it is computationally demanding, but we implemented on-demand audio loading: the playback button can be clicked, it will become gray while the audio is loaded, and start playback and transform into a pause button. This avoids downloading large files for users who are only tagging visually (e.g., for bat recordings). We have restored the functionality for returning to the previous spectrogram view with an instant back browser button press. However, we decided to keep the time and frequency indications inside the selection boxes only instead of adding scales, as we find that information redundant and haven't found a best practice implementation yet (but see issue \#162). Finally, our new collection search function instantly searches across all the files' meta-data and does not require a search button press.

Competing Interests: No competing interests were disclosed.

The benefits of publishing with F1000Research:

- Your article is published within days, with no editorial bias

- You can publish traditional articles, null/negative results, case reports, data notes and more

- The peer review process is transparent and collaborative

- Your article is indexed in PubMed after passing peer review

- Dedicated customer support at every stage

For pre-submission enquiries, contact research@f1000.com 